\title{
Hypoxia enhances the induction of human amniotic mesenchymal side population cells into vascular endothelial lineage
}

\author{
NAOKO MARUYAMA ${ }^{1}$, KENICHI KOKUBO $^{1,2}$, TOSHIHIRO SHINBO ${ }^{2}$, MINORU HIROSE $^{1,2}$, \\ MAMORU KOBAYASHI $^{2}$, NORIO SAKURAGAWA ${ }^{2}$ and HIROSUKE KOBAYASHI ${ }^{1,2}$ \\ ${ }^{1}$ Graduate School of Medical Sciences; ${ }^{2}$ School of Allied Health Sciences, Kitasato University, Sagamihara, Japan
}

Received November 19, 2012; Accepted February 4, 2013

DOI: $10.3892 / \mathrm{ijmm} .2013 .1412$

\begin{abstract}
Human amniotic mesenchymal side population (hAM-SP) cells have pluripotency and weak immunogenicity, and have promising roles in the field GAPDH of regenerative medicine. The aim of the present study was to determine whether hypoxic conditions induce the differentiation of hAM-SP cells into the vascular endothelial lineage. Mesenchymal cells were isolated from enzyme-treated amniotic membranes and stained with Hoechst 33342. The hAM-SP cells were negatively sorted by FACS and cultured in induction medium containing vascular endothelial growth factor (VEGF) under normoxic $\left(20 \% \mathrm{O}_{2}\right)$ or hypoxic $\left(1 \% \mathrm{O}_{2}\right)$ conditions for 1 or 2 weeks. The expression of endothelial markers such as kinase domain region (KDR), fms-like tyrosine kinase (Flt)-1, von Willebrand factor (vWF), vascular endothelial (VE)-cadherin and human vascular cell adhesion molecule (VCAM) at the gene and protein level was evaluated by real-time PCR and fluorescent immunostaining, respectively. The gene expression of KDR, Flt-1, VE-cadherin and vWF peaked after 2 weeks of culture. The protein expression of KDR and VE-cadherin was also enhanced after 2 weeks of culture under hypoxic conditions. To confirm the involvement of hypoxia-inducible factor (HIF) in the induction under hypoxic conditions, the expression of genes which are known to be upregulated by HIF was analyzed by DNA microarray. The expression of these genes increased under hypoxic conditions. hAM-SP cells cultured under hypoxic conditions differentiated into the vascular endothelial lineage, probably due to upregulation of the gene expression associated with angiogenesis through activation of the HIF system.
\end{abstract}

\section{Introduction}

Human amniotic mesenchymal cells (hAMCs), which are derived from amniotic membranes, are an attractive stem

Correspondence to: Dr Kenichi Kokubo, Department of Medical Engineering and Technology, School of Allied Health Sciences, Kitasato University, 1-15-1 Kitasato, Minami-ku, Sagamihara, Kanagawa 252-0373, Japan

E-mail: kokubo@kitasato-u.ac.jp

Key words: hypoxia, amniotic mesenchymal stem cell, side population, vascular endothelial lineage, hypoxia-inducible factor cell source in the field of regenerative medicine (1). hAMCs have weak immunogenicity due to their negligible expression of human leukocyte antigen (HLA) class II molecules, low expression levels of HLA class I molecules (2), and high expression levels of immunosuppressive factors, including interleukin-1 receptor antagonist (IL-1ra), IL-10 and collagen XVIII $(3,4)$. There are relatively fewer ethical constraints in using hAMCs, since hAMCs are obtained from the amnion, which is discarded after childbirth. Since the amniotic membrane is derived from the inner cell mass of the blastocyst and is of fetal origin, it is expected that amniotic cells contain pluripotent stem cells. Amniotic epithelial and mesenchymal cells express POU domain class 5 transcription factor 1 (Oct-3/4, ES cell makers), nestin and musashi (neural stem cell markers) (5), suggesting that the cells derived from the amniotic membranes indeed contain undifferentiated cells.

Several methods have been used to induce the differentiation of hAMCs into endothelial cells. When hAMCs were cultured in a medium appropriate for endothelial cell culture (EGM-2 $2^{\mathrm{TM}}$ medium) containing hydrocortisone, human epidermal growth factor (hEGF), fetal bovine serum (FBS), vascular endothelial growth factor (VEGF), human fibroblast growth factor-basic (hFGF-B), the cells changed in morphology from fibroblast-like shape to endothelial cell-like shape, and they also developed the ability to take up acetylated low-density lipoprotein (LDL) and form endothelial-like networks in the Matrigel ${ }^{\mathrm{TM}}$ assay (6). VEGF has also been shown to induce hAMCs to differentiate into endothelial cells. When the cells were cultured on Matrigel ${ }^{\mathrm{TM}}$, spontaneous differentiation of hAMCs into endothelial cells was also detected. VEGF was shown to enhance the expression of fms-like tyrosine kinase (Flt)-1 and kinase domain region (KDR) in hAMCs, and to also induce the expression of endothelial cell-specific markers such as intercellular adhesion molecule (ICAM)-1, CD34 and von Willebrand factor (vWF) (7). Human amniotic fluid-derived cells have also been used as a source of mesenchymal stem cells. The cells acquired endothelial cell characteristics when cultured in EGM-2 medium or under a shear force created by setting the culture dish on an orbital shaker, and to produce angiogenic factors such as VEGF, placental growth factor (PGF) and hepatocyte growth factor (HGF) when cultured under hypoxic conditions $\left(5 \% \mathrm{O}_{2}\right)(8)$. 
Angiogenesis is induced by hypoxia in several situations, such as in the development of the retinal circulation in premature infants $(9)$, wound repair $(10,11)$, and tumor angiogenesis $(12,13)$. The hypoxia-inducible factor (HIF) system plays an important role in the regulation of angiogenesis under hypoxic conditions. In the presence of hypoxia, HIF upregulates VEGF transcription $(14,15)$, as well as the expression of two VEGF receptors, Flt-1 (16) and KDR (17), which increase the biological activity of secreted VEGF.

Cells that are negatively stained by Hoechst 33342 (DNA-binding fluorescent dye), i.e., cells with the highest efflux capacity for the dye, are known to be a very small and homogeneous population of highly primitive cells, known as side population (SP) cells (18-21). SP cells found in a number of species, including mice, monkeys and humans, and isolated from several organs, including the bone marrow, skeletal muscle and liver (18-21), have demonstrated the potential for differentiation into cell types beyond their organ of origin (22). SP cells have also been isolated from hAMCs (23). hAM-SP cells express Oct-3/4 and have the potential to differentiate into multiple lineages, including several organ- or tissuespecific cells including neurons, osteoblasts, chondrocytes, and adipocytes, as found for the other type of mesenchymal stem cells (23).

The aim of the present study was to clarify whether hAM-SP cells, which can be regarded as an undifferentiated stem cell fraction of AMCs, were effectively induced to differentiate into cells of endothelial lineage by hypoxia. Therefore, we cultured hAM-SP cells in an endothelial induction medium containing VEGF in a hypoxic $\left(1 \% \mathrm{O}_{2}\right)$ or a normoxic $\left(20 \% \mathrm{O}_{2}\right)$ environment.

\section{Materials and methods}

Preparation of human amniotic mesenchymal side population cells. The Institutional Ethics Committee approved all protocols (Kitasato University, School of Allied Health Sciences, no. 2009-015). The protocol for the preparation of amniotic mesenchymal SP cells has previously been described (23). Briefly, after informed consent was obtained from a pregnant woman scheduled for caesarean section, the amniotic membrane was separated from the post-partum placenta. The human amniotic membrane consists of two cell layers, the epithelial layer and mesenchymal layer, with the basement membrane between the two. To prepare AMCs, the amniotic membrane was first treated with trypsin to remove the amniotic epithelial cells, and then the remnant layer was treated with an enzyme mixture $(0.1 \%$ papain, $1 \mathrm{mg} / \mathrm{ml}$ collagenase, $0.01 \%$ DNase, and $0.1 \%$ dispase) to dissolve the mesenchymal layer and disperse cells. The AMCs were stained with Hoechst 33342 and the SP cells were sorted using a cell sorter (EPICS Altra; Beckman Coulter, Fullerton, CA, USA).

The sorted SP cells in the hAMCs (hAM-SP cells) were cultured in Dulbecco's modified Eagle's medium nutrient mixture F-12 Ham (DMEM/F12) containing 5\% FBS (both were from Sigma-Aldrich, St. Louis, MO, USA), $10 \mathrm{ng} / \mathrm{ml}$ human leukemia inhibitory factor (hLIF; Chemicon-Merck Millipore, Billerica, MA, USA), $10 \mathrm{ng} / \mathrm{ml}$ hFGF-B (PeproTech, Inc., Rocky Hill, NJ, USA) and $10 \mathrm{ng} / \mathrm{ml}$ platelet-derived growth factor-BB (PDGF-BB; PeproTech, Inc.), on a type I collagen-coated dish (Iwaki, Chiba, Japan) in a $5 \% \mathrm{CO}_{2}$ environment at $37^{\circ} \mathrm{C}$. The cells were cultured until they reached $90 \%$ confluence and were recovered with $0.1 \%$ trypsin (SigmaAldrich)-ethylenediaminetetraacetic acid (EDTA; Gibco-Life Technologies, Carlsbad, CA, USA), and sub-cultured at a density of $10^{4}$ cells $/ \mathrm{cm}^{2}$.

Endothelial differentiation culture. The hAM-SP cells at the fourth or fifth passages cultured in cell growth medium were rinsed with phosphate-buffered saline (PBS) without $\mathrm{Ca}^{2+}$ and $\mathrm{Mg}^{2+}$ and then cultured in endothelial induction medium consisting of DMEM/F12 supplemented with $2 \%$ FBS and $50 \mathrm{ng} / \mathrm{ml}$ VEGF (PeproTech, Inc.) for 1 or 2 weeks in a hypoxic $\left(1 \% \mathrm{O}_{2}\right)$ or normoxic $\left(20 \% \mathrm{O}_{2}\right)$ environment, on a type I collagen-coated dish. As control, the hAM-SP cells were cultured in normal medium consisting of DMEM/F12 supplemented with $2 \%$ FBS in the absence of VEGF for 1 or 2 weeks. The endothelial induction medium and normal medium were replenished every two days. The endothelial markers after differentiation of the hAM-SP cells were evaluated by real-time PCR and fluorescence immunostaining.

Real-time PCR. To examine the expression of endothelial cell-specific mRNA, we performed a quantitative reverse transcription-polymerase chain reaction (qRT-PCR) assay. RNAprotect ${ }^{\circledR}$ Cell Reagent (Qiagen, Hilden, Germany) was used to stabilize the RNA of the cultivated cells and total RNA was extracted from the cells using RNeasy Plus Mini kit (Qiagen). The mRNA was transcribed into cDNA using the iScript $^{\mathrm{TM}}$ cDNA Synthesis kit (Bio-Rad, Hercules, CA, USA). PCR was carried out by mixing $1 \mu \mathrm{l}$ of cDNA template, each primer (Table I) and SYBR-Green (Bio-Rad) in a volume of $20 \mu 1$. The samples were amplified in a thermocycler. Each sample was analyzed in triplicate by the Chromo 4 system (Bio-Rad). Amplification data were obtained using the software of Opticon Monitor (Bio-Rad). The expression levels were quantified relative to the expression level of GAPDH.

Immunochemistry. hAM-SP cells subjected to induction were fixed and incubated for $1 \mathrm{~h}$ with diluted primary antibodies specific for the endothelial cells. The primary antibodies used as endothelial markers were: anti-human vascular cell adhesion molecule (VCAM)-1 mouse IgG (1:100; Immunotech, Beckman Coulter, Inc.), anti-human KDR mouse IgG (1:100; Sigma-Aldrich), anti-human vascular endothelial (VE)-cadherin mouse IgG (1:100; R\&D Systems, Inc., Minneapolis, MN, USA), and anti-human vWF mouse IgG (1:50; Santa Cruz Biotechnology, Inc., Santa Cruz, CA, USA). The secondary antibodies were Alexa Fluor 488-conjugated anti-mouse goat IgG (1:500; Molecular Probes-Life Technologies). The immunostained cells were analyzed by confocal laser scanning microscopy (CLSM) (IX70; Olympus Corporation, Tokyo, Japan).

Expression of the Oct-3/4 protein was evaluated by fluorescent immunostaining following induction with VEGF under hypoxic conditions for 1 or 2 weeks. The primary antibody used was anti-human Oct-3/4 rabbit IgG (1:200; Santa Cruz Biotechnology, Inc.), and the secondary antibody was Alexa 488-conjugated anti-rabbit goat IgG (1:500; Molecular 
Table I. Primer sets used for RT-PCR analysis of the endothelial differentiation.

Forward primers

Reverse primers

$\begin{array}{ll}\text { GAPDH } & \text { 5'-GGCC TCCA AGGA GTAA GACC-3' } \\ \text { KDR } & \text { 5'-AGCC AGCT CTGG ATTT GTGG A-3' } \\ \text { Flt-1 } & \text { 5'-GCGC TTCA CCTG GACT GACA-3' } \\ \text { VCAM-1 } & \text { 5'-ATTG ACTT GCAG CACC ACAG-3' } \\ \text { vWF } & \text { 5'-AGAT GTTT GCCT ACGG CTTG-3' }\end{array}$

5'-AGGG GTCT ACAT GGCA ACTG-3'

5'-CATG CCCT TAGC CACT TGGA A-3'

5'-GAAA CTGG GCCT GCTG ACAT C-3'

5'-ATCT CCAG CCTG TCAA ATGG-3'

5'-CAGC CTGT GACC CTCT TCTC-3'

RT-PCR, reverse transcription-polymerase chain reaction; KDR, kinase domain region; Flt-1, fms-like tyrosine kinase-1; VCAM-1, vascular cell adhesion molecule-1; vWF, von Willebrand factor.

Probes). Immunostained cells were analyzed by confocal laser microscopy using a CLSM. Each RGB image was separated into red, green and blue by color deconvolution with the ImageJ 1.40 software (National Institutes of Health, Bethesda, $\mathrm{MD}, \mathrm{USA}$ ), and the numbers of pixels in green color (endothelial markers) and red color (Oct-3/4) were calculated.

Microarray processing. All experiments were performed using commercially available microarrays for humans (Human Genome U133 Plus 2.0 Array, Affymetrix, Santa Clara, CA, USA). The hAM-SP cells cultivated with VEGF under hypoxic $\left(1 \% \mathrm{O}_{2}\right)$ conditions for 2 or 0 weeks were removed from the culture dishes using trypsin and washed with PBS. Total RNA was isolated from the cells using an RNeasy Mini $\mathrm{kit}^{\circledR}$ (Qiagen). The isolation and purification of the total RNA were carried out according to the manufacturer's protocol (Qiagen). The quality and amount of starting RNA were confirmed by agarose gel electrophoresis in addition to the ratio of absorbance $\left(1.9<\mathrm{A}_{260 \mathrm{~nm}} / \mathrm{A}_{280 \mathrm{~nm}}<2.0\right)$. Total RNA was used to prepare a biotinylated target cRNA according to the manufacturer's recommendation (Affymetrix). Briefly, $1 \mu \mathrm{g}$ of mRNA was used to generate the first-strand cDNA using a T7-linked oligo(dT) primer. After second-strand synthesis, in vitro transcription was performed using a synthetic biotinylated nucleotide analog (biotinylated uridine-triphosphate), which yielded an 50-100-fold amplification of RNA. The cRNA was fragmented prior to overnight hybridization. The arrays were then washed, stained with streptavidin-phycoerythrin, and scanned (GeneChip Scanner $3000^{\circledR}$; Affymetrix).

After scanning, the array images were visually inspected to confirm the scanner alignment and the absence of significant bubbles or scratches on the chip surface. The $3^{\prime} / 5^{\prime}$ ratios for GAPDH were 1.34 and 1.63 , which were within the acceptable limits; BioB spike controls were also present on all the chips, with BioC, BioD and Cre being present in increasing intensities. BioB, BioC, BioD and Cre are genes from Escherichia coli or bacteriophage P1 and were added before hybridization to check the hybridization quality. Background intensities were 93.8 and 102.6 and noise factors were 5.08 and 5.12, which were sufficiently low.

Statistical analysis. All data are presented as the means + SD. Bonferroni's post hoc test was used for multiple-group comparisons. P-values $<0.05$ were considered to indicate statistically significant differences.

\section{Results}

For evaluation of the endothelial differentiation potency, the gene expression of endothelial markers such as KDR (24), Flt-1 (25), VCAM (26) and vWF (27) was evaluated by realtime PCR. While the expression of KDR, VCAM and vWF did not change after 1 week of culture with VEGF under hypoxic conditions, the expression of Flt-1 increased (Fig. 1).

After 2 weeks of induction with VEGF under hypoxic conditions (Fig. 2), the hAM-SP cells showed enhanced expression of KDR, Flt-1, VCAM and vWF. The cells cultivated only under hypoxic conditions in the absence of VEGF or the cells induced with VEGF under normoxic conditions showed no significant changes in the expression of genes as compared to those in the control (cultivated without VEGF under normoxic conditions). On the other hand, the cells induced with VEGF under hypoxic conditions showed significantly increased expression levels of KDR, VCAM, Flt-1 and vWF as compared to the cells induced with VEGF under normoxic conditions as well as to the cells cultured only under hypoxic conditions in the absence of VEGF or the cells induced with VEGF under normoxic conditions (Fig. 2). Thus, culture under hypoxic conditions enhanced the cellular differentiation by VEGF into the endothelial lineage.

Immunocytochemical assay for endothelial markers such as VCAM, KDR, vWF and VE-cadherin (28) was performed following induction of hAM-SP cells with VEGF under normoxic/hypoxic conditions for 2 weeks. Positive staining for KDR and VE-cadherin proteins was observed following induction with VEGF under both normoxic and hypoxic conditions, while staining for the VCAM and vWF proteins was negative (Fig. 3A). Human pulmonary artery endothelial cells (HPAECs) were used as positive control, and showed positive staining for all markers (data not shown). The number of pixels in green color was calculated with the ImageJ software (Fig. 3B). KDR was significantly induced by VEGF under both normoxic and hypoxic conditions as compared with that in the control conditions $(\mathrm{P}<0.01)$. VE-cadherin expression was increased by VEGF under normoxic conditions as compared with that in the control conditions $(\mathrm{P}<0.05)$, and was induced even more significantly under hypoxic condition as compared with that in normoxic conditions $(\mathrm{P}<0.01)$. To evaluate if any undifferentiated cells remained after the induction, immunocytochemical staining for Oct-3/4 was carried out after induction with VEGF under hypoxic condi- 

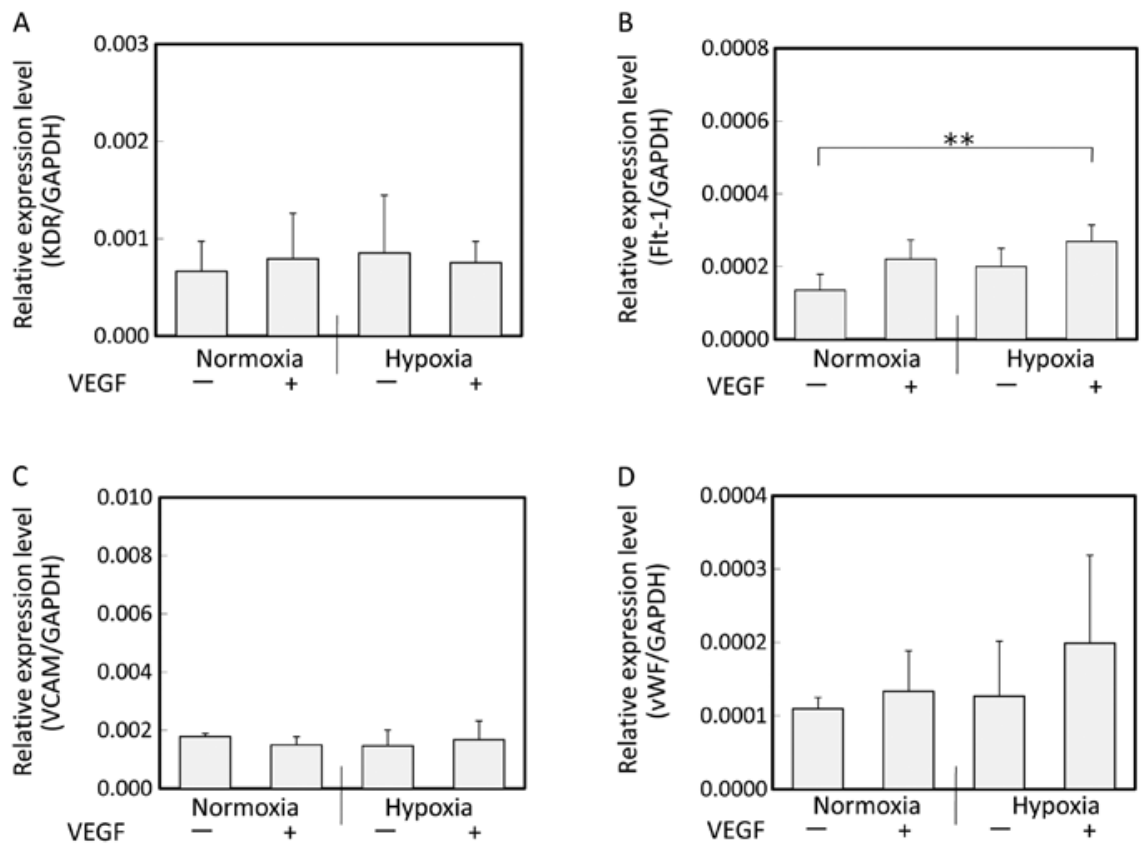

Figure 1. Gene expression of endothelial cell markers evaluated by real-time PCR after 1 week of induction with VEGF. (A) KDR, (B) Flt-1, (C) VCAM, (D) vWF. Cells were induced by VEGF under normoxic or hypoxic conditions. The expression levels relative to the GAPDH expression level were compared. While no changes in the expression of KDR, VCAM or vWF were observed after induction with VEGF under hypoxic conditions, the expression of Flt-1 increased. Data shown are the means + SD $(\mathrm{n}=5) ;\left({ }^{* *} \mathrm{P}<0.01\right)$. VEGF, vascular endothelial growth factor; KDR, kinase domain region; Flt-1, fms-like tyrosine kinase-1; VCAM, vascular cell adhesion molecule; vWF, von Willebrand factor.

A
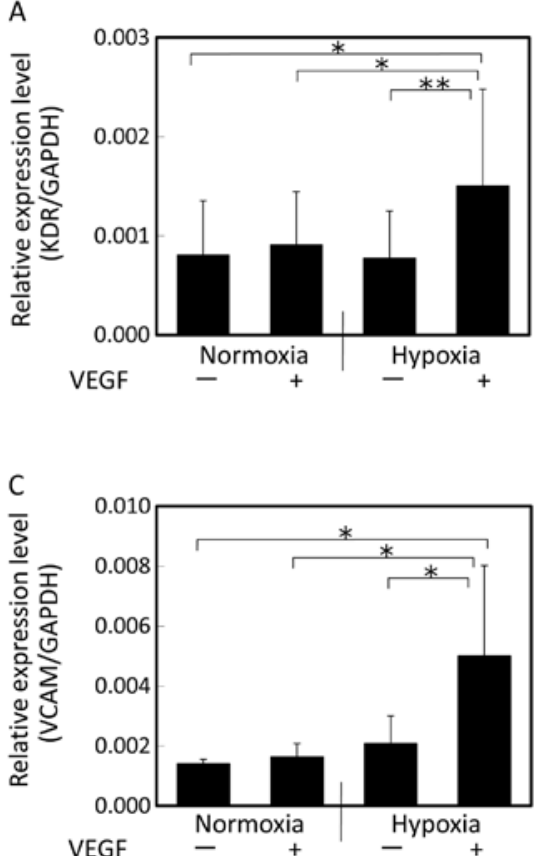
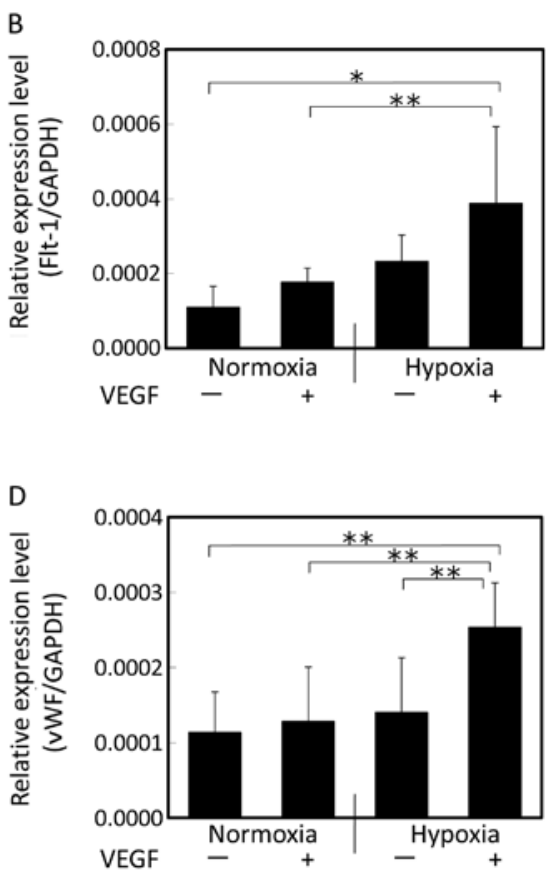

Figure 2. Gene expression of endothelial cell markers evaluated by real-time PCR after 2 weeks of induction with VEGF. (A) KDR, (B) Flt-1, (C) VCAM, (D) vWF. Cells were induced by VEGF under normoxic or hypoxic conditions. The expression levels relative to the GAPDH expression level were compared. Following cultivation under hypoxic conditions in the presence of VEGF, significant increase in the expression levels of KDR, Flt-1, VCAM and vWF was observed. Data shown are the means $+\mathrm{SD}(\mathrm{n}=5) ;\left({ }^{*} \mathrm{P}<0.05\right.$ and $\left.{ }^{* *} \mathrm{P}<0.01\right)$. VEGF, vascular endothelial growth factor; KDR, kinase domain region; Flt-1, fms-like tyrosine kinase-1; VCAM, vascular cell adhesion molecule; vWF, von Willebrand factor.

tions for 1 or 2 weeks (Fig. 4). Strong expression of Oct-3/4, which is a marker gene of undifferentiated cells $(29,30)$, was observed in the hAM-SP cells prior to the induction. While some expression of Oct-3/4 protein was still observed after
1 week of induction with VEGF under hypoxic conditions, the red staining for this protein disappeared almost entirely after 2 weeks of induction. The expression of Oct-3/4 protein decreased significantly with the induction time. 
A
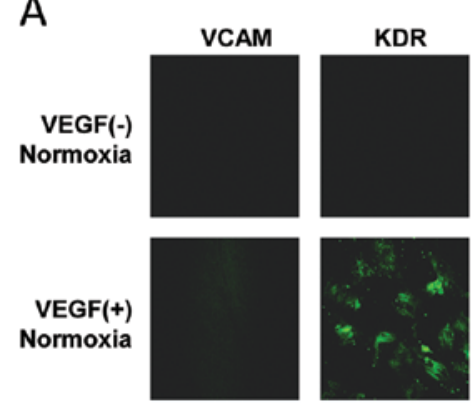

VEGF(+)

Hypoxia
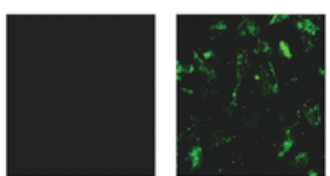
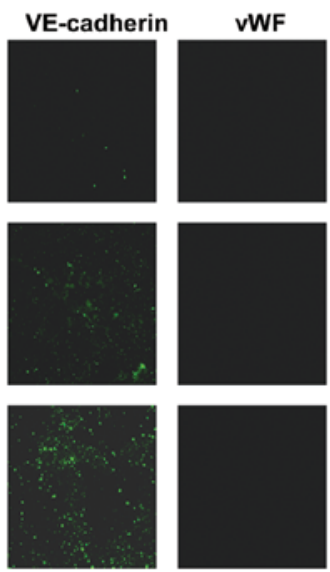

B

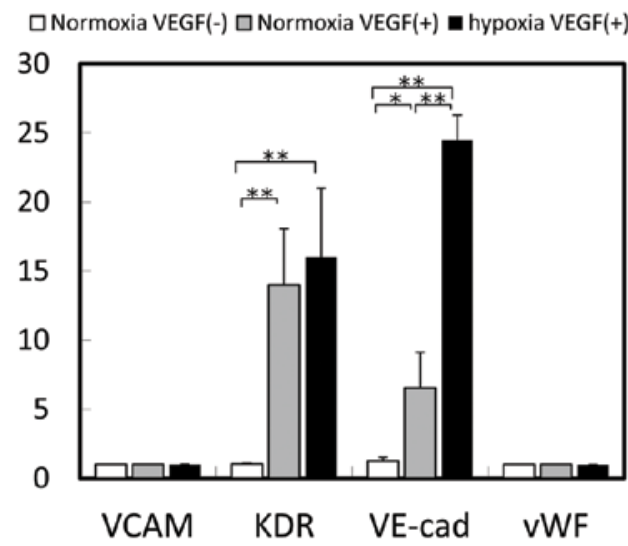

Figure 3. (A) Fluorescent immunostaining for endothelial cell markers following induction with VEGF for 2 weeks under hypoxic or normoxic conditions. The primary antibodies used were: anti-human VCAM mouse IgG, anti-human Flk-1 mouse IgG and anti-human VE-cadherin mouse IgG, and the secondary antibodies were Alexa Fluor 488-conjugated anti-mouse goat IgG. (B) The number of pixels in green color extracted by color deconvolution was calculated with the ImageJ software. Data shown are the means $+\mathrm{SD}(\mathrm{n}=4) ;\left(\mathrm{P}<0.05\right.$ and $\left.{ }^{* * *} \mathrm{P}<0.01\right)$. KDR expression was significantly increased by cultivation with VEGF under both normoxic as well as hypoxic conditions. Staining for VE-cadherin was stronger following cultivation under hypoxic than normoxic conditions. Negative staining was found for VCAM and vWF. VEGF, vascular endothelial growth factor; VCAM, vascular cell adhesion molecule; KDR, kinase domain region; vWF, von Willebrand factor.
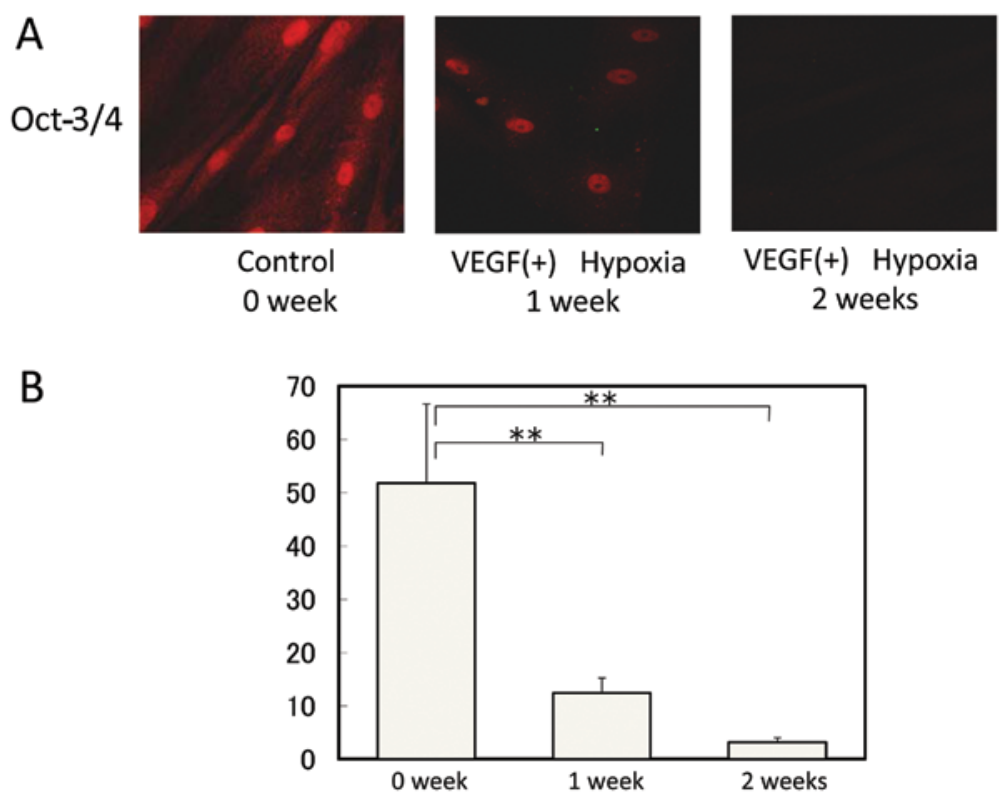

Figure 4. (A) Fluorescent immunostaining for Oct-3/4 following induction with VEGF for 1 or 2 weeks under hypoxic conditions. The primary antibody used was anti-human Oct-3/4 rabbit IgG, and the secondary antibody was Alexa 488-conjugated anti-rabbit goat IgG. The immunostained cells were analyzed by confocal laser scanning microscopy. The control group showed positive staining for Oct-3/4; Oct-3/4 expression was still observed after 1 week of induction under hypoxic conditions, while it was no longer detected after 2 weeks of induction. (B) The number of pixels in red color was calculated with the ImageJ software. Data shown are the means $+\mathrm{SD}(\mathrm{n}=4) ;\left({ }^{* *} \mathrm{P}<0.01\right)$. VEGF, vascular endothelial growth factor.

We also analyzed gene expression by microarray analysis and focused on the gene expression of stem cell markers (31) and neural stem cell markers. The gene expression of stem cell markers, such as Nanog homeobox (NANOG) $(32,33)$, Oct-3/4 (29,30) and growth differentiation factor 3 (GDF3) (34), was decreased by less than half in the cells cultivated with VEGF under hypoxic conditions for 2 weeks as compared to those cultivated for 0 weeks (prior to induction). The gene expression of Kruppel-like factor 4 (KLF4) (35), v-myc myelocytomatosis viral oncogene homolog (MYC) (36), sex determining region Y (SRY)-box 2 (SOX2) (37), RNA exonuclease 1 homolog (REX1) (38), fibroblast growth factor 4 (FGF4) (39), telomerase reverse transcriptase (TERT) (40) slightly decreased (or remained the same) following induction with VEGF under hypoxic conditions for 2 weeks. The neural stem cell markers such as nestin (41) and musashi (42) were decreased after induction with VEGF under hypoxic conditions for 2 weeks (Table II).

To confirm the involvement of HIF in the induction under hypoxic conditions, we selected downstream genes of HIF-1. The results revealed that the gene expression of VEGF-A (15), Flt-1 (16), erythropoietin (EPO) (43), enolase 
Table II. Gene expression of stem cell markers and neural stem cell markers by microarray analysis.

\begin{tabular}{|c|c|c|c|}
\hline \multirow[b]{2}{*}{ Gene } & \multicolumn{3}{|c|}{ Hypoxia VEGF(+) } \\
\hline & Detection & 2/0 week & Author/(ref.) \\
\hline \multicolumn{4}{|l|}{ Stem cell markers } \\
\hline Kruppel-like factor 4 (gut) (KLF4) & $\mathrm{P} / \mathrm{P}$ & 0.98 & Li et al (35) \\
\hline v-myc myelocytomatosis viral oncogene homolog (MYC) & $\mathrm{P} / \mathrm{P}$ & 0.88 & Cartwright et al (36) \\
\hline Nanog homeobox (NANOG) & $\mathrm{A} / \mathrm{A}$ & 0.08 & $\begin{array}{l}\text { Chambers et al (32) } \\
\text { Mitsui et al (33) }\end{array}$ \\
\hline POU domain class 5 transcription factor 1 (Oct-3/4) & $\mathrm{A} / \mathrm{A}$ & 0.47 & $\begin{array}{l}\text { Nichols et al (29) } \\
\text { Niwa et al (30) }\end{array}$ \\
\hline Sex determining region Y (SRY)-box 2 (SOX2) & $\mathrm{A} / \mathrm{A}$ & 1.00 & Avilion et al (37) \\
\hline RNA exonuclease 1 homolog (REX1) & $\mathrm{A} / \mathrm{A}$ & 0.83 & Ben-Shushan et al (38) \\
\hline Growth differentiation factor 3 (GDF3) & $\mathrm{A} / \mathrm{A}$ & 0.24 & Levine and Brivanlou (34) \\
\hline Fibroblast growth factor 4 (FGF4) & $\mathrm{A} / \mathrm{A}$ & 0.81 & Yuan et al (39) \\
\hline Telomerase reverse transcriptase (TERT) & $\mathrm{P} / \mathrm{P}$ & 0.67 & Yang et al (40) \\
\hline \multicolumn{4}{|l|}{ Neural stem cell markers } \\
\hline Nestin (NES) & $\mathrm{P} / \mathrm{A}$ & 0.09 & Park et al (41) \\
\hline Musashi homolog 1 (MSI1) & $\mathrm{A} / \mathrm{A}$ & 0.71 & Kaneko et al (42) \\
\hline
\end{tabular}

Detection indicates whether the transcript was present $(\mathrm{P})$ or absent $(\mathrm{A})$ calls. Present and absent calls mean that the expression levels are above or below the threshold of detection.

Table III. Hypoxia-inducible factor-1 target genes upregulated by hypoxic culture.

\begin{tabular}{lccc}
\hline Gene & Detection & $\begin{array}{c}\text { Hypoxia VEGF(+) } \\
\text { 2/0 week }\end{array}$ & Author/(ref.) \\
\hline Vascular endothelial growth factor A (VEGF-A) & $\mathrm{P} / \mathrm{P}$ & 2.0 & Forsythe et al (15) \\
Fms-like tyrosine kinase-1 (flt-1) & $\mathrm{P} / \mathrm{P}$ & 2.7 & Gerber et al $(16)$ \\
Erythropoietin (EPO) & $\mathrm{A} / \mathrm{A}$ & 6.6 & Wang and Semenza (43) \\
Enolase 1 (ENO-1) & $\mathrm{P} / \mathrm{P}$ & 2.0 & Semenza et al $(44)$ \\
Adrenomedullin (ADM) & $\mathrm{P} / \mathrm{P}$ & 6.3 & Nguyen and Claycomb (45) \\
EGL nine homolog 3 (EGLN-3) & $\mathrm{P} / \mathrm{P}$ & 17.9 & Pescador et al (46)
\end{tabular}

Detection indicates whether the transcript was present $(\mathrm{P})$ or absent $(\mathrm{A})$ calls. Present and absent calls mean that the expression levels are above or below the threshold of detection.

(ENO)-1 (44), adrenomedullin (ADM) (45) and Egl nine homolog (EGLN)-3 (46), which are known to be upregulated by HIF-1, were increased by more than 2-fold in the cells cultivated with VEGF under hypoxic conditions for 2 weeks as compared to those cultivated with VEGF under normoxic conditions (Table III).

\section{Discussion}

To clarify whether hAM-SP cells can be effectively induced to differentiate into endothelial lineage cells by hypoxia, hAM-SP cells were cultured in endothelial cell induction medium containing VEGF under hypoxic $\left(\begin{array}{ll}1 \% & \mathrm{O}_{2}\end{array}\right)$ or normoxic $\left(20 \% \mathrm{O}_{2}\right)$ conditions. Our data revealed that under hypoxic conditions: i) the gene expression of endothelial lineage markers such as KDR, Flt-1, VCAM and vWF was induced; ii) the expression of endothelial marker proteins including KDR and VE-cadherin was induced; and iii) expression of the HIF target genes was upregulated in the hAM-SP cells. Cultivation in the presence of VEGF under hypoxic conditions for 2 weeks enhanced the expression of endothelial lineage markers. While the expression of Oct-3/4 was still observed after 1 week of induction with VEGF under hypoxic conditions, it disappeared almost completely after 2 weeks. These results suggest that induction of hAM-SP cells for 1 week was inadequate to induce differentiation of the cells into endothelial cells, while induction for 2 weeks led to pronounced differentiation of the cells into the endothelial lineage. However, protein expression of VCAM and vWF could not be detected under these conditions. Therefore, the characteristics of hAM-SP cells following induction by VEGF under hypoxic condition for 2 weeks were not entirely identical to those of endothelial cells, and it is suggested that extended culture time would have created more mature endo- 
thelial cells, since enhanced mRNA expression of vWF and VCAM was observed.

hAMCs have been reported to be induced by EGM-2, which contain several growth factors, including VEGF; however, the differentiated cells did not express mature endothelial cell markers, such as vWF and VE-cadherin (6). Also, VEGF receptors 1 (Flt-1) and 2 (KDR) were basally expressed in hAMCs, and enhanced expression of endothelial-specific markers such as Flt-1 KDR and ICAM-1 was observed following exposure to VEGF (7). These results indicate that while hAMCs may have the potential to differentiate into endothelial cells, they are a heterogeneous population of cells. hAM-SP cells have a larger population of undifferentiated cells, while the hAMCs contain these cells only at the rate of $0.1-0.2 \%$. In the present study conducted using hAM-SP cells, low expression levels of KDR and Flt-1 in the hAM-SP cells and a high expression level of Oct-3/4 were observed prior to induction. The gene expression of KDR, Flt-1 and vWF, as well as the protein expressions of VE-cadherin increased following induction with VEGF under hypoxic conditions. hAM-SP cells include several stem cells in a more pluripotent state than non-SP cells, and are, therefore, preferable as the source of cells for use in the field of regenerative medicine.

Bone marrow-derived mesenchymal stem cells (BM-MSCs) have also been used as candidate cells in the field of regenerative medicine. Reduced oxygen tension in the physiological range (4-7\%) (47) has been shown to enhance the proliferation of these cells. Attention has been focused on the effects of hypoxia on the differentiation of pluripotent cells into various mesenchymal lineages. Although the exact outcome depended on the oxygen tension, time in culture, and use/non-use of hypoxic preculture, the beneficial effects appeared more often on osteogenic, chondrogenic and adipogenic differentiation (48). Although the target cells of these studies were not endothelial cells, they did show that hypoxia clearly plays an important role in the differentiation of mesenchymal stem cells. Hypoxia was also found to be an important factor inducing the differentiation of hAM-SP cells into the endothelial lineage.

It is well-known that VEGF transcription is upregulated under hypoxic conditions by the effects of HIF-1. HIF-1 also upregulates two VEGF receptors, Flt-1 (16) and KDR (17), which increase the biological activities of secreted VEGF (49). To confirm the involvement of HIF in the effective induction of hAM-SP cells under hypoxic conditions, we analyzed the changes in the expression of downstream genes of HIF-1. Our results revealed that the gene expression of VEGF-A, Flt-1, EPO, ENO-1, ADM and EGLN-3, which are known to be upregulated by HIF, $(15,16,43-46)$ increased following induction with VEGF under hypoxic conditions for 2 weeks. These results indicate that under hypoxic conditions, the HIF system is activated, which enhances the expressions of VEGF and VEGF receptors leading to the differentiation into the endothelial lineage.

The hypoxic environment (3-5\% of oxygen) is physiologically normal for embryonic stem cells and its pluripotency is regulated by the family of HIFs (50). From the microarray data and Oct-3/4 staining, stem cell markers are gradually decreased by induction of VEGF under hypoxic conditions. These results indicate that hypoxic conditions would also contribute to maintaining an undifferentiated state of hAM-SP cells and maximizing the differentiation into vascular endothelial cells by VGEF by suppressing the differentiation into the other types of cells.

In conclusion, the hAM-SP cells cultivated in the presence of VEGF under hypoxic conditions differentiated into the vascular endothelial lineage, possibly due to upregulation of the gene expression associated with angiogenesis through activation of the HIF system.

\section{References}

1. Sakuragawa N, Yoshikawa $\mathrm{H}$ and Sasaki M: Amniotic tissue transplantation: clinical and biochemical evaluations for some lysosomal storage diseases. Brain Dev 14: 7-11, 1992.

2. Wolbank S, Peterbauer A, Fahrner M, et al: Dose-dependent immunomodulatory effect of human stem cells from amniotic membrane: a comparison with human mesenchymal stem cells from adipose tissue. Tissue Eng 13: 1173-1183, 2007.

3. Hori J, Wang M, Kamiya K, Takahashi H and Sakuragawa N: Immunological characteristics of amniotic epithelium. Cornea 25 (Suppl 1): S53-S58, 2006.

4. Lefebvre S, Adrian F, Moreau P, et al: Modulation of HLA-G expression in human thymic and amniotic epithelial cells. Hum Immunol 61: 1095-1101, 2000.

5. Sakuragawa N, Kakinuma K, Kikuchi A, et al: Human amnion mesenchyme cells express phenotypes of neuroglial progenitor cells. J Neurosci Res 78: 208-214, 2004.

6. Konig JM, Huppertz B, Desoye G, et al: Amnion-derived mesenchymal stromal cells show angiogenic properties but resist differentiation into mature endothelial cells. Stem Cells Dev 21: 1309-1320, 2012

7. Alviano F, Fossati V, Marchionni C, et al: Term amniotic membrane is a high throughput source for multipotent mesenchymal stem cells with the ability to differentiate into endothelial cells in vitro. BMC Dev Biol 7: 11, 2007.

8. Zhang P, Baxter J, Vinod K, Tulenko TN and Di Muzio PJ: Endothelial differentiation of amniotic fluid-derived stem cells: synergism of biochemical and shear force stimuli. Stem Cells Dev 18: 1299-1308, 2009.

9. Ashton N, Ward B and Serpell G: Effect of oxygen on developing retinal vessels with particular reference to the problem of retrolental fibroplasia. Br J Ophthalmol 38: 397-432, 1954.

10. Knighton DR, Silver IA and Hunt TK: Regulation of woundhealing angiogenesis-effect of oxygen gradients and inspired oxygen concentration. Surgery 90: 262-270, 1981.

11. Knighton DR, Hunt TK, Scheuenstuhl H, Halliday BJ, Werb Z and Banda MJ: Oxygen tension regulates the expression of angiogenesis factor by macrophages. Science 221: 1283-1285, 1983 .

12. Thomlinson RH and Gray LH: The histological structure of some human lung cancers and the possible implications for radiotherapy. Br J Cancer 9: 539-549, 1955.

13. Folkman J, Merler E, Abernathy C and Williams G: Isolation of a tumor factor responsible for angiogenesis. J Exp Med 133: 275-288, 1971.

14. Liu Y, Cox SR, Morita T and Kourembanas S: Hypoxia regulates vascular endothelial growth factor gene expression in endothelial cells. Identification of a 5' enhancer. Circ Res 77: 638-643, 1995.

15. Forsythe JA, Jiang BH, Iyer NV, et al: Activation of vascular endothelial growth factor gene transcription by hypoxia-inducible factor 1. Mol Cell Biol 16: 4604-4613, 1996.

16. Gerber HP, Condorelli F, Park J and Ferrara N: Differential transcriptional regulation of the two vascular endothelial growth factor receptor genes. Flt-1, but not Flk-1/KDR, is up-regulated by hypoxia. J Biol Chem 272: 23659-23667, 1997.

17. Waltenberger J, Mayr U, Pentz S and Hombach V: Functional upregulation of the vascular endothelial growth factor receptor KDR by hypoxia. Circulation 94: 1647-1654, 1996.

18. Goodell MA, Rosenzweig M, Kim H, et al: Dye efflux studies suggest that hematopoietic stem cells expressing low or undetectable levels of CD34 antigen exist in multiple species. Nat Med 3: 1337-1345, 1997.

19. Welm BE, Tepera SB, Venezia T, Graubert TA, Rosen JM and Goodell MA: Sca-1(pos) cells in the mouse mammary gland represent an enriched progenitor cell population. Dev Biol 245: 42-56, 2002. 
20. Wulf GG, Luo KL, Jackson KA, Brenner MK and Goodell MA: Cells of the hepatic side population contribute to liver regeneration and can be replenished with bone marrow stem cells. Haematologica 88: 368-378, 2003.

21. Uchida N, Fujisaki T, Eaves AC and Eaves CJ: Transplantable hematopoietic stem cells in human fetal liver have a CD34(+) side population (SP)phenotype. J Clin Invest 108: 1071-1077, 2001 .

22. Gussoni E, Soneoka Y, Strickland CD, et al: Dystrophin expression in the mdx mouse restored by stem cell transplantation. Nature 401: 390-394, 1999.

23. Kobayashi M, Yakuwa T, Sasaki K, et al: Multilineage potential of side population cells from human amnion mesenchymal layer. Cell Transplant 17: 291-301, 2008.

24. Yamaguchi TP, Dumont DJ, Conlon RA, Breitman ML and Rossant J: flk-1, an flt-related receptor tyrosine kinase is an early marker for endothelial cell precursors. Development 118 : 489-498, 1993.

25. Shibuya M, Seetharam L, Ishii Y, et al: Possible involvement of VEGF-FLT tyrosine kinase receptor system in normal and tumor angiogenesis. Princess Takamatsu Symp 24: 162-170, 1994.

26. Lobb R, Chi-Rosso G, Leone D, et al: Expression and functional characterization of a soluble form of vascular cell adhesion molecule 1. Biochem Biophys Res Commun 178: 1498-1504, 1991.

27. Jones TR, Kao KJ, Pizzo SV and Bigner DD: Endothelial cell surface expression and binding of factor VIII/von Willebrand factor. Am J Pathol 103: 304-308, 1981.

28. Lampugnani MG, Resnati M, Raiteri M, et al: A novel endothelial-specific membrane protein is a marker of cell-cell contacts. J Cell Biol 118: 1511-1522, 1992.

29. Nichols J, Zevnik B, Anastassiadis K, et al: Formation of pluripotent stem cells in the mammalian embryo depends on the POU transcription factor Oct4. Cell 95: 379-391, 1998.

30. Niwa H, Miyazaki J and Smith AG: Quantitative expression of Oct-3/4 defines differentiation, dedifferentiation or self-renewal of ES cells. Nat Genet 24: 372-376, 2000.

31. Imamura M, Miura K, Iwabuchi K, et al: Transcriptional repression and DNA hypermethylation of a small set of ES cell marker genes in male germline stem cells. BMC Dev Biol 6: 34, 2006.

32. Chambers I, Colby D, Robertson M, et al: Functional expression cloning of Nanog, a pluripotency sustaining factor in embryonic stem cells. Cell 113: 643-655, 2003.

33. Mitsui K, Tokuzawa Y, Itoh H, et al: The homeoprotein Nanog is required for maintenance of pluripotency in mouse epiblast and ES cells. Cell 113: 631-642, 2003.

34. Levine AJ and Brivanlou AH: GDF3, a BMP inhibitor, regulates cell fate in stem cells and early embryos. Development 133 209-216, 2006

35. Li Y, McClintick J, Zhong L, Edenberg HJ, Yoder MC and Chan RJ: Murine embryonic stem cell differentiation is promoted by SOCS-3 and inhibited by the zinc finger transcription factor Klf4. Blood 105: 635-637, 2005.
36. Cartwright P, McLean C, Sheppard A, Rivett D, Jones K and Dalton S: LIF/STAT3 controls ES cell self-renewal and pluripotency by a Myc-dependent mechanism. Development 132: 885-896, 2005

37. Avilion AA, Nicolis SK, Pevny LH, Perez L, Vivian N and LovellBadge R: Multipotent cell lineages in early mouse development depend on SOX2 function. Genes Dev 17: 126-140, 2003.

38. Ben-Shushan E, Thompson JR, Gudas LJ and Bergman Y: Rex-1, a gene encoding a transcription factor expressed in the early embryo, is regulated via Oct-3/4 and Oct- 6 binding to an octamer site and a novel protein, Rox-1, binding to an adjacent site. Mol Cell Biol 18: 1866-1878, 1998.

39. Yuan H, Corbi N, Basilico C and Dailey L: Developmentalspecific activity of the FGF-4 enhancer requires the synergistic action of Sox2 and Oct-3. Genes Dev 9: 2635-2645, 1995.

40. Yang C, Przyborski S, Cooke MJ, et al: A key role for telomerase reverse transcriptase unit in modulating human embryonic stem cell proliferation, cell cycle dynamics, and in vitro differentiation. Stem Cells 26: 850-863, 2008.

41. Park D, Xiang AP, Mao FF, et al: Nestin is required for the proper self-renewal of neural stem cells. Stem Cells 28: 2162-2171, 2010.

42. Kaneko Y, Sakakibara S, Imai T, et al: Musashi1: an evolutionally conserved marker for CNS progenitor cells including neural stem cells. Dev Neurosci 22: 139-153, 2000

43. Wang GL and Semenza GL: Characterization of hypoxia-inducible factor 1 and regulation of DNA binding activity by hypoxia. J Biol Chem 268: 21513-21518, 1993.

44. Semenza GL, Roth PH, Fang HM and Wang GL: Transcriptional regulation of genes encoding glycolytic enzymes by hypoxiainducible factor 1. J Biol Chem 269: 23757-23763, 1994.

45. Nguyen SV and Claycomb WC: Hypoxia regulates the expression of the adrenomedullin and HIF-1 genes in cultured HL-1 cardiomyocytes. Biochem Biophys Res Commun 265: 382-386, 1999.

46. Pescador N, Cuevas Y, Naranjo S, et al: Identification of a functional hypoxia-responsive element that regulates the expression of the egl nine homologue 3 (egln3/phd3) gene. Biochem J 390: 189-197, 2005.

47. Lennon DP, Edmison JM and Caplan AI: Cultivation of rat marrow-derived mesenchymal stem cells in reduced oxygen tension: Effects on in vitro and in vivo osteochondrogenesis. J Cell Physiol 187: 345-355, 2001.

48. Das R, Jahr H, van Osch GJ and Farrell E: The role of hypoxia in bone marrow-derived mesenchymal stem cells: considerations for regenerative medicine approaches. Tissue Eng Part B Rev 16: 159-168, 2010

49. Pugh CW and Ratcliffe PJ: Regulation of angiogenesis by hypoxia: role of the HIF system. Nat Med 9: 677-684, 2003.

50. Szablowska-Gadomska I, Zayat V and Buzanska L: Influence of low oxygen tensions on expression of pluripotency genes in stem cells. Acta Neurobiol Exp 71: 86-93, 2011. 